\title{
The neuropsychopharmacology of neuropathic pain
}

\author{
Harold Merskey DM FRCP FRCPC FRCPsych
}

$\mathrm{T}^{\mathrm{h}}$ his series of papers provides a review of recent advances in understanding and managing neuropathic pain. The papers arise from consideration of epidemiological issues (Morley-Forster, pages 5A-10A), the complexities of the distinction between inflammatory and neuropathic pain (Bennett, pages 11A-15A), systematic consideration of efficacy and safety issues related to gabapentin and pregabalin (Gilron and Flatters, pages 16A-29A), and clinical management of neuropathic pain (Moulin, pages 30A-36A).

In this special supplement, Dr Morley-Forster, looking at the epidemiology of neuropathic pain, uses Bennett's definition of neuropathic pain as "pain due to a primary lesion of the peripheral or central nervous system" (1). Dr Moulin, by contrast, uses the definition of the International Association for the Study of Pain (2): "pain initiated or caused by a primary lesion or dysfunction of the central nervous system".

Because the topic under consideration is essentially pain due to nerve damage, Dr Morley-Forster can be comfortable, and so can the reader, with the definition she selected. The survey of pain due to nerve damage is instructive, and the more variable and less distinct syndromes such as complex regional pain syndrome are important, but information on them is less sound in any case. The survey tells us that the prevalence of neuropathic pain, even narrowly defined, is substantial and likely to increase, partly because of advances in medical treatment that enable many patients to survive, albeit at a cost in ancillary ill effects.

Dr Moulin, on the other hand, uses the broader definition, which can encompass migraine and other conditions in which dysfunction is evident but anatomical change is less certain. For example, hypocalcemia leading to tetany or, a much more common problem, migraine with aura, both reflect a dysfunction that may be due to chemical influences or vascular components and ultimately may lead to tissue damage of one sort or another, but also that is less readily definable for the purposes of therapeutic pharmacology. Each, then, uses the definition that suits a relevant purpose, although, of course, I would prefer the broader definition with a comment, when relevant, that the restricted part of the definition is being applied.

There is a lesson here for taxonomy and perhaps the International Association for the Study of Pain definition of neuropathic pain should be amended to suggest that there are two major divisions, one with a neuroanatomical foundation and the other with a chemical or pathophysiological basis. Broad distinctions of this type become paradoxically less persuasive and more acceptable in light of Dr Bennett's discussion of inflammatory and neuropathic pain. His dissection of the similarities and differences of these phenomena, currently regarded as separate, leads to a new potential framework for physiological classifications of types of pain. It also leads me to conclude that, for practical purposes, we can legitimately indicate whether we are discussing the pathoanatomic type of neuropathy or the purely dysfunctional version, or both. After that, we can, as he says, consider whether we are going to be able to differentiate, for example, inflammatory cutaneous mechanoallodynia from neuropathic cutaneous mechanoallodynia.

Other issues arise with psychopharmacology and neuropharmacology. We have begun to realize that the neurological and psychotropic medications affecting sensory status and the contents of consciousness are often unpredictable. Even a drug that is recognized consistently as an antidepressant, such as amitriptyline, may produce a dysphoric reaction in 5\% to $10 \%$ of patients who first take it. This is different from merely disliking the commonplace side effects of constipation or dry mouth, while appreciating that the drug has sedative, analgesic and antidepressant actions. It amounts to actually experiencing a degree of subjective distress from the treatment that was intended to relieve sadness. This is true in one way or another, to greater or lesser extents, for all or most antidepressants. The monoamine oxidase inhibitors can give rise to such a subjective state, although they are generally less prone to do so than most of the tricyclic antidepressants. In turn, the more recent antidepressants can activate thought in a way which may be dangerous. Increased suicidal ideation after taking paroxetine (3) is the same phenomenon that was recognized to occur spontaneously in patients with recovering depression before the days of antidepressants, but correctly draws to our attention the fact that antidepressants may increase suicidal ideas. Other stimulant antidepressants (such as bupropion or amphetamines) may stimulate unpleasantly rather than happily. With respect to opioids and cannabinoids, some patients certainly like the sedative, calming and analgesic effects of opioids, but others may find them unpleasant, arousing in a way that is not comfortable or causing feelings of distress more than pain relief.

The variety of effects that can be obtained with the same drug, sometimes acting in different directions on the nervous system, may not, after all, be surprising. A serotoninergic drug may influence one serotoninergic receptor and not another. The last time I counted (several years ago), there were 
upwards of 12 known different serotonin receptors, and the count was still rising. There are more serotonin receptors than serotoninergic drugs, and each of the latter has a slightly different spectrum of action. If we consider the many different types of receptors known in the nervous system, whether they be the relatively few that seem to be related to cholinergic mechanisms, the several that are related to adrenergic mechanisms, the increasing number of dopaminergic receptors or the rising numbers of recognized opioid receptors, the idea that all drugs directed to a type of receptor have only one or two effects seems unlikely. Perhaps fortunately, most pharmacological preparations for the nervous system seem to affect more than one receptor or receptor type. This may be desirable in view of the many thousands of genes, each of which may be responsible for a different receptor and require a suitable remedy when its effects are disruptive or insufficient.

Many prescribers have abandoned the notion that it is bad to use more than one drug for a single disorder. Hypertension is typically controlled with two or three different medications as required, rather than with a large dose of a single agent; small doses of each of several medications may be much better tolerated and more useful. The old fashioned pharmacology, which physicians may still practise and with which many grew up, emphasized the importance of not using more than one drug for one situation. That view has become untenable, although the alternative view - many drugs in small doses for each illness - still encounters resistance.

Many drugs have more than one action - perhaps particularly so in the case of the central nervous system. Amytriptyline was demonstrated to have an analgesic effect in the absence of depression (4). Anticonvulsants are easily recognized as drugs for the nervous system that have long been used for more than one purpose. Phenobarbitone was used for epilepsy and nocturnal sedation, and to calm agitated individuals. The use of carbamazepine in the treatment of trigeminal neuralgia and also diabetic neuropathy dates back at least as far as the 1960s (5). In addition, these drugs and their younger relations, such as valproate, topiramate and lamotrigine, are used for the control of bipolar illness as effective alternatives to lithium carbonate. Topiramate has been put forward as helpful for headache, especially migraine. Level I evidence and clinical experience are held to support the use of divalproates and topiramate, together with amitriptyline and beta-blockers, in the prevention of migraine. We are awash with options. Even so, the gabapentinoids have established a prominent position in the treatment of at least certain types of neuropathic pain (6), as shown here in the extensive review by Drs Gilron and Flatters. Interestingly, pregabalin has been reported, in a conventional double-blind, placebo-controlled trial, to be effective for fibromyalgia (7), although this is not a Canadian indication. This raises the interesting question as to whether the central sensitization phenomena of fibromyalgia are the point of effect for the medication. That remains to be seen, because pregabalin also has anxiolytic effects, and anxiety is common in clinic populations with fibromyalgia and may theoretically contribute to the increase in pain.

Altogether, we have some interesting substances. It seems likely that no one medication is going to sweep the field and cure all patients with chronic pain, but every additional useful drug that does not have serious side effects is likely to help more patients than would get better by chance and to make a contribution to the important, but still relatively neglected, field of the relief of pain.

\section{REFERENCES}

1. Bennett GJ. Neuropathic pain: A crisis of definition? Anesth Analg 2003;97:619-20.

2. Merskey H, Bogduk N. Classification of Chronic Pain. Descriptions of Chronic Pain Syndromes and Definitions of Pain Terms. Seattle: IASP Press, 1994.

3. Healy D. Let Them Eat Prozac: The Unhealthy Relationship Between the Pharmaceutical Industry and Depression. New York: New York University Press, 2004.

4. Watson CP, Evans RJ, Reed K, Merskey H, Goldsmith L, Warsh J. Amitriptyline versus placebo in postherpetic neuralgia. Neurology 1982;32:671-3.

5. Rull JA, Quibrera R, Gonzalez-Millan H, Lozano Castaneda O. Symptomatic treatment of peripheral diabetic neuropathy with carbamazepine (Tegretol). Double blind cross-over trial. Diabetologia 1969;5:215-8.

6. Buchanan TM, Ramadan NM. Prophylactic pharmacotherapy for migraine headaches. Semin Neurol 2006;26:188-98.

7. Crofford LJ, Rowbotham MC, Mease PJ, et al; Pregabalin 1008-105 Study Group. Pregabalin for the treatment of fibromyalgia syndrome: Results of a randomized, double-blind, placebocontrolled trial. Arthritis Rheum 2005;52:1264-73. 


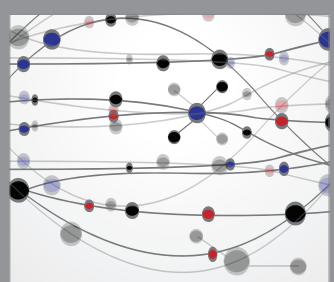

The Scientific World Journal
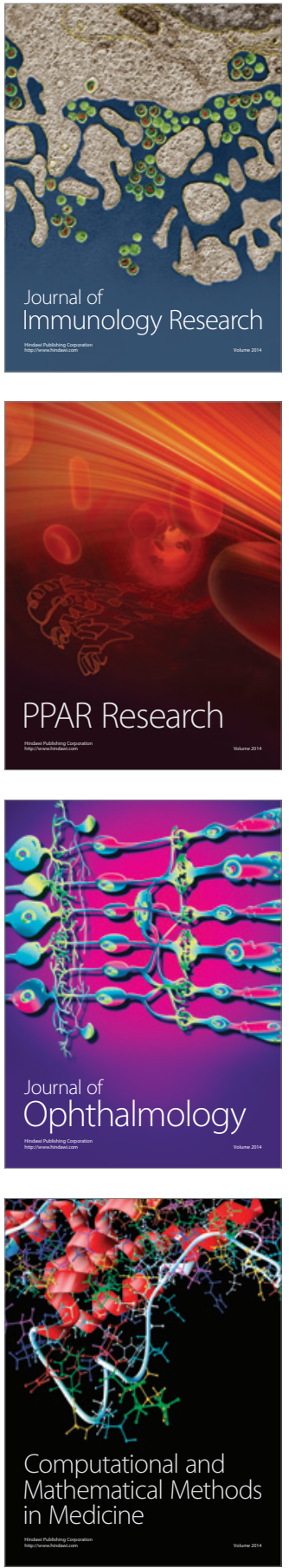

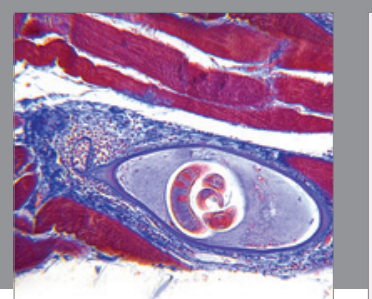

Gastroenterology Research and Practice

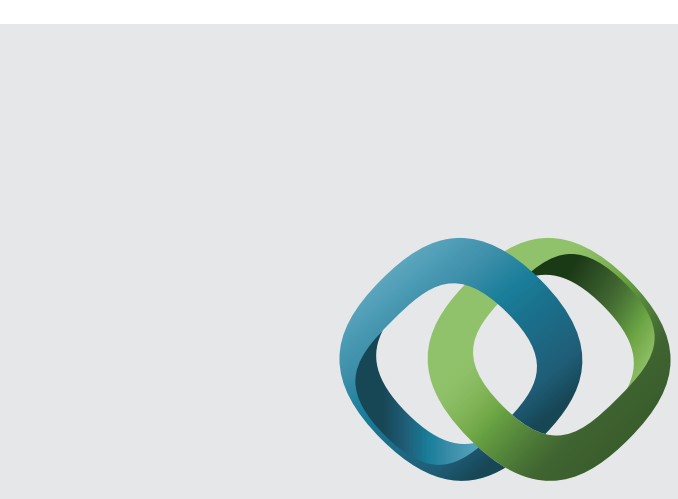

\section{Hindawi}

Submit your manuscripts at

http://www.hindawi.com
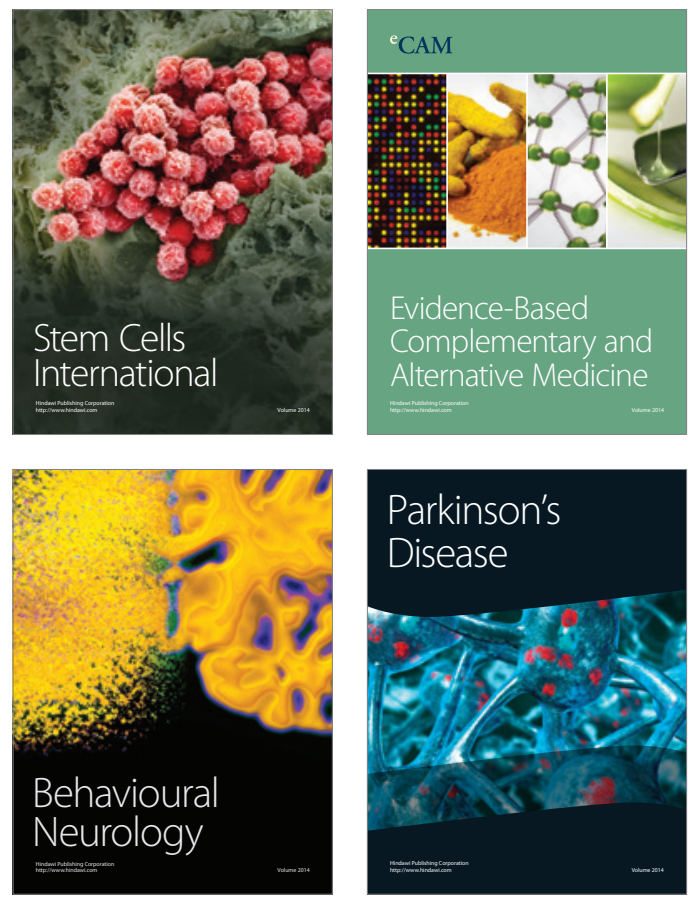
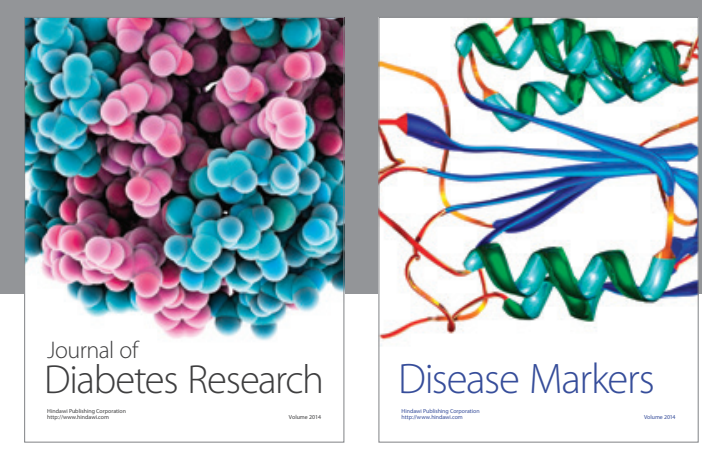

Disease Markers
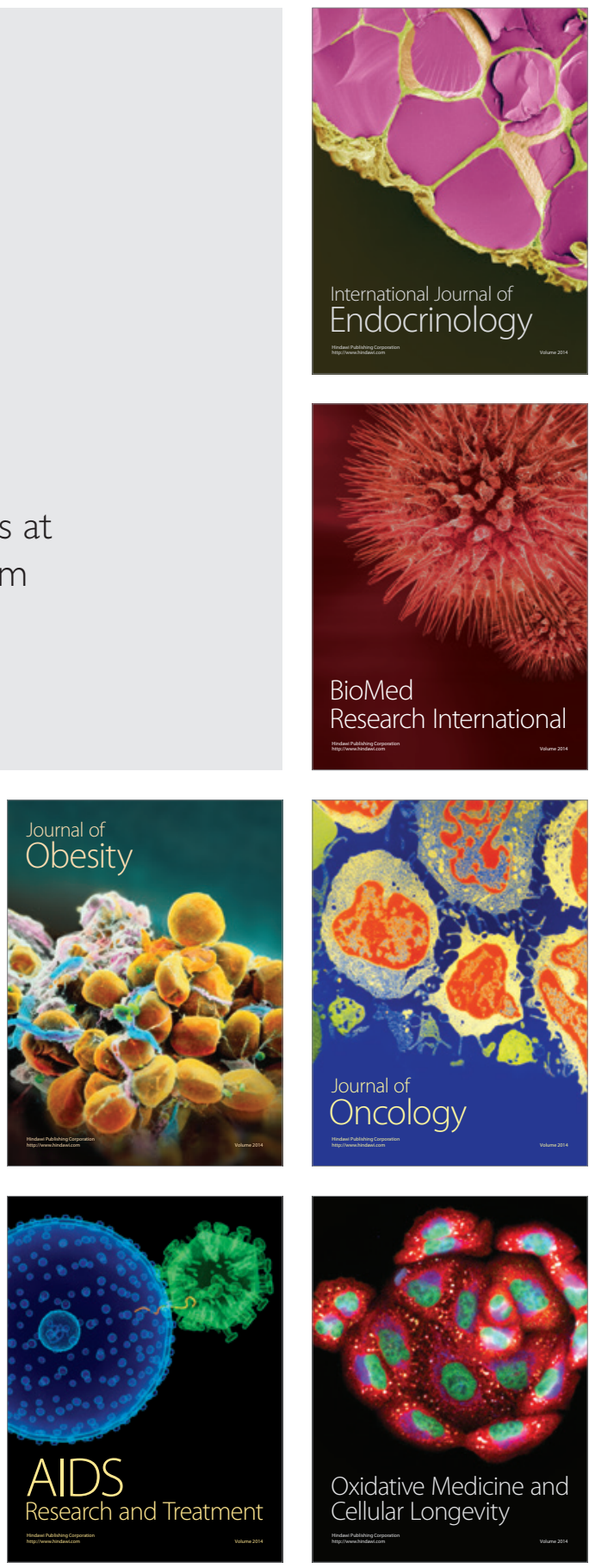